\title{
Cosmic rays and stochastic magnetic reconnection in the heliotail
}

\author{
P. Desiati ${ }^{1,2}$ and A. Lazarian ${ }^{2}$ \\ ${ }^{1}$ Wisconsin IceCube Particle Astrophysics Center, University of Wisconsin, Madison, WI 53706, USA \\ ${ }^{2}$ Dept. of Astronomy, University of Wisconsin, Madison, WI 53706, USA \\ Correspondence to: P. Desiati (desiati@icecube.wisc.edu), A. Lazarian (lazarian@astro.wisc.edu)
}

Received: 28 September 2011 - Revised: 15 May 2012 - Accepted: 16 May 2012 - Published: 19 June 2012

\begin{abstract}
Galactic cosmic rays are believed to be generated by diffusive shock acceleration processes in Supernova Remnants, and the arrival direction is likely determined by the distribution of their sources throughout the Galaxy, in particular by the nearest and youngest ones. Transport to Earth through the interstellar medium is expected to affect the cosmic ray properties as well. However, the observed anisotropy of $\mathrm{TeV}$ cosmic rays and its energy dependence cannot be explained with diffusion models of particle propagation in the Galaxy. Within a distance of a few parsec, diffusion regime is not valid and particles with energy below about $100 \mathrm{TeV}$ must be influenced by the heliosphere and its elongated tail. The observation of a highly significant localized excess region of cosmic rays from the apparent direction of the downstream interstellar flow at $1-10 \mathrm{TeV}$ energies might provide the first experimental evidence that the heliotail can affect the transport of energetic particles. In particular, $\mathrm{TeV}$ cosmic rays propagating through the heliotail interact with the 100$300 \mathrm{AU}$ wide magnetic field polarity domains generated by the $11 \mathrm{yr}$ cycles. Since the strength of non-linear convective processes is expected to be larger than viscous damping, the plasma in the heliotail is turbulent. Where magnetic field domains converge on each other due to solar wind gradient, stochastic magnetic reconnection likely occurs. Such processes may be efficient enough to re-accelerate a fraction of $\mathrm{TeV}$ particles as long as scattering processes are not strong. Therefore, the fractional excess of $\mathrm{TeV}$ cosmic rays from the narrow region toward the heliotail direction traces sightlines with the lowest smearing scattering effects, that can also explain the observation of a harder than average energy spectrum.
\end{abstract}

\section{Introduction}

During the last decades, galactic cosmic rays have been found to have a small but measurable energy dependent uneven arrival direction distribution, with a relative amplitude of order $10^{-4}-10^{-3}$. This anisotropy was observed in the Northern Hemisphere from energies of tens to several hundreds $\mathrm{GeV}$ with muon detectors (Nagashima et al., 1998; Munakata et al., 2010), and in the multi-TeV energy range with Tibet AS $\gamma$ array (Amenomori et al., 2006, 2011a), Super-Kamiokande (Guillian et al., 2007), Milagro (Abdo et al., 2009) and ARGO-YBJ (Zhang, 2009; Shuwang, 2011). An anisotropy was also observed at an energy in excess of about $100 \mathrm{TeV}$ with the EAS-TOP shower array (Aglietta et al., 2009). Recently similar observations were reported in the Southern Hemisphere at energies of $10 \mathrm{~s}$ to $100 \mathrm{~s} \mathrm{TeV}$ with the IceCube Observatory (Abbasi et al., 2010, 2012). While at $\sim 10 \mathrm{TeV}$ the anisotropy appears to be topologically connected to the GeV-TeV observations in the north above about $100 \mathrm{TeV}$, the global anisotropy persists but with a different phase consistent with the results from Aglietta et al. (2009). The top panel of Fig. 1 shows the combined map in equatorial coordinates of relative intensity of cosmic ray arrival direction distribution observed by Tibet AS $\gamma$ at about $5 \mathrm{TeV}$ in the Northern Hemisphere, and by IceCube at about $20 \mathrm{TeV}$ in the Southern Hemisphere.

The arrival distribution of sub- $\mathrm{TeV}$ cosmic rays revealed the existence of two kinds of anisotropies, as discussed in $\mathrm{Na}-$ gashima et al. (1998). One is a global non-dipolar anisotropy with relative excess approximately centered around equatorial right ascension of $0 \mathrm{hr}$, and an increasing amplitude up to $1-10 \mathrm{TeV}$ energies. The other is a directional excess region confined in a cone of half opening angle of $68^{\circ}$ from right ascension of about $6 \mathrm{~h}$, and observed for energies below $\mathrm{TeV}$. This region covers a portion of the sky that includes 

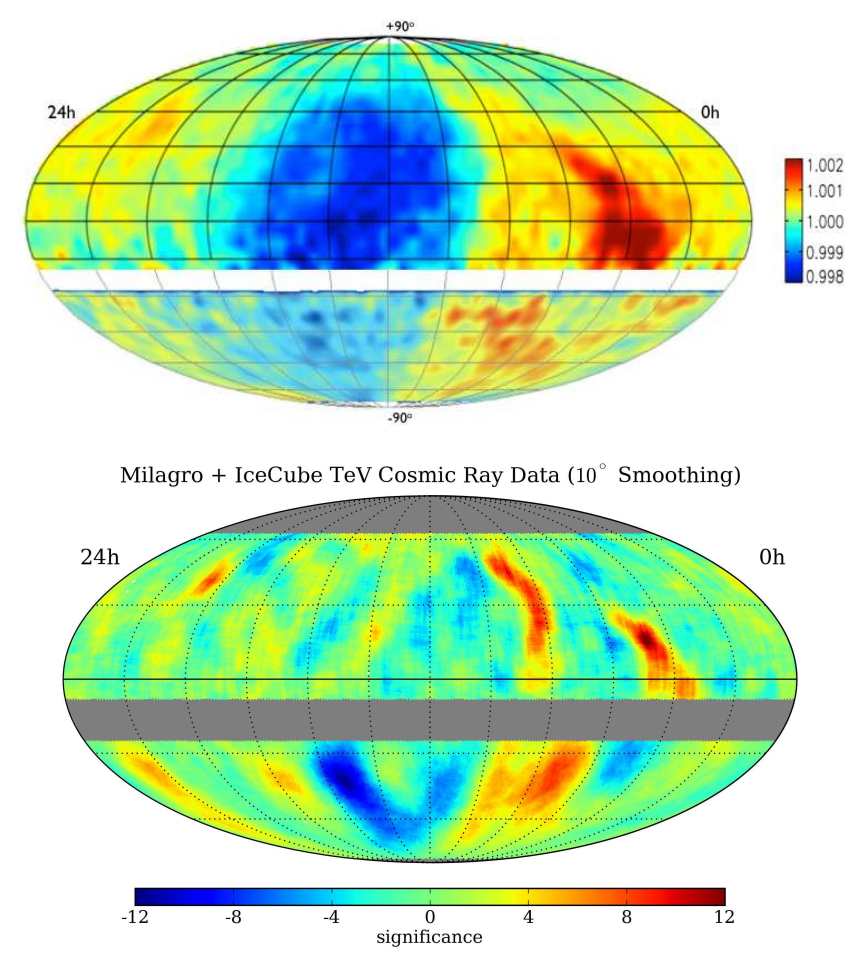

Fig. 1. Top panel: map in equatorial coordinates of the relative intensity of the cosmic ray arrival distribution as observed by the Tibet $\mathrm{AS} \gamma$ at about $5 \mathrm{TeV}$ in the Northern Hemisphere (from Amenomori et al., 2011a), and by the IceCube Observatory at a median energy of $20 \mathrm{TeV}$ in the Southern Hemisphere (from Abbasi et al., 2010). Bottom panel: map in equatorial coordinates of the statistical significance of the cosmic ray arrival direction distribution as observed by Milagro at about $1 \mathrm{TeV}$ in the Northern Hemisphere (from Abdo et al., 2008), and by the IceCube Observatory at a median energy of $20 \mathrm{TeV}$ in the Southern Hemisphere (from Abbasi et al., 2011). In this map features with angular extension larger than $30-60^{\circ}$ are filtered out.

the direction of the heliospheric tail (or heliotail), which is the region of the heliosphere downstream from the interstellar wind delimited within the heliopause, i.e. the boundary that separates the solar wind and the interstellar plasmas (Izmodenov and Kallenbach, 2006). Its origin was therefore attributed to some unidentified anisotropic process occurring in the heliotail, and thus it was called "tail-in" excess.

At the higher $\mathrm{TeV}$ energies, while the tail-in broad excess becomes sub-dominant, the global anisotropy shows evidence of statistically significant small angular structures from the same direction in the sky. In particular, using experimental techniques in the attempt to isolate relatively localized excess or deficit regions of events that overlap over the smooth global anisotropy, angular features of order $20-30^{\circ}$ were discovered. Two separate, highly significant, localized, fractional, excess regions of cosmic rays were reported in the Northern Hemisphere by Milagro (Abdo et al., 2008), and also by Tibet AS $\gamma$ (Amenomori et al., 2007) and ARGO-YBJ
(Vernetto et al., 2009; Iuppa, 2011). The observation of a small scale anisotropy at multi-TeV energies was reported by IceCube, in the Southern Hemisphere, as well (Abbasi et al., 2011). The bottom panel of Fig. 1 shows the combined map in equatorial coordinates of statistical significance of the cosmic ray arrival direction distribution where only features with angular extension smaller than about $60^{\circ}$ are visible. Such small scale features lay in the same portion of the sky where the tail-in excess was dominant at lower energy, especially the one toward the heliotail direction with equatorial coordinates $(\alpha, \delta) \approx\left(5 \mathrm{~h},+17^{\circ}\right)$.

At an energy in excess of about $100 \mathrm{TeV}$, where the anisotropy has a different topology than at lower energy, cosmic ray particles are hardly influenced by the heliosphere and its elongated tail, and their arrival direction might hold information on the Local Interstellar Magnetic Field (LIMF) on a larger scale. If the extended heliotail induces a significant perturbation in the local interstellar medium, that can affect the arrival direction of multi- $\mathrm{TeV}$ cosmic ray particles, then the anisotropy can be considered as an indirect probe of how the LIMF influences the heliospheric boundary itself (see Desiati and Lazarian, 2011). Moreover, cosmic rays below about $10 \mathrm{TeV}$ are expected to be influenced by magnetic fields inside the heliotail as well. The concurrent effects of magnetic reconnection and scattering processes might be able to explain some observations, although more experimental results and further developments in heliospheric Magneto Hydro-Dynamic (MHD) simulations are needed for better constrain models.

The origin of the cosmic ray anisotropy, its persistence in a wide energy range and its angular structure, is currently subject of debate. In this paper we will briefly report the interpretations provided by various authors (in Sect. 2), with an emphasis on a possible phenomenological connection between the broad tail-in excess of sub-TeV cosmic rays and the localized fractional excess of multi- $\mathrm{TeV}$ cosmic rays from the direction of the heliotail. We will then describe the magnetic field structure in the heliotail as shaped by solar cycles and rotation in Sect. 3. An overview on magnetic reconnection processes is given in Sect. 4, with an emphasis on stochastic reconnection, assumed to contribute to the origin of the anomalies observed toward the heliotail. Section 5 addresses the proposed mechanism of cosmic ray re-acceleration in the heliotail and its effective influence in relation to scattering processes.

\section{Cosmic rays anisotropy}

The origin of cosmic ray anisotropy is still unknown. The relative motion of the solar system, with respect to the cosmic ray plasma rest frame (for instance due to galactic rotation), would produce a dipolar anisotropy in the direction of the motion (Compton and Getting, 1935; Gleeson and Axford, 1968). Such Compton-Getting effect was not singled 
out from observations yet, inducing to a possible conclusion that the bulk of galactic cosmic rays co-rotates with the solar system (Amenomori et al., 2006). Moreover, in the scenario where galactic cosmic rays are accelerated in supernova remnants, their arrival direction should have a relative excess toward the galactic center, i.e. the line of sight with the larger expected number of sources. On the other hand the nearest galactic sources would dominate the observed arrival distribution, and changes in anisotropy amplitude and phase with cosmic ray energy can arise as a natural consequence of the stochastic nature of their sources in the local interstellar medium (Erlykin and Wolfendale, 2006; Blasi and Amato, 2012).

Propagation properties of cosmic rays in the interstellar medium are likely to have an important role in shaping the anisotropy as well (Battaner et al., 2009). For instance, a scenario where the large scale anisotropy is linked to diffusion of cosmic rays through the LIMF connecting the solar system to the interstellar medium outside the local interstellar cloud (where the solar system currently resides) was proposed by Amenomori et al. (2007, 2011b). This model accounts for the apparent quadrupolar contribution observed with the large scale anisotropy. In Frisch (2011) it is noted that the tail-in excess region, besides including the heliotail direction, is centered around the direction of the LIMF, therefore linking its origin to their propagation deep inside the tail or to streaming along the LIMF or the S1 sub-shell of Loop I superbubble.

Within a distance of a few times the mean free path, diffusion regime breaks down and propagation of cosmic rays depends on their interaction with the turbulence ripples of the LIMF. Even though observations suggest that the LIMF is coherent over scales of about $100 \mathrm{pc}$, they also imply variations in field directions of less than $30-40^{\circ}$, that can be attributed to turbulence (Frisch, 2011). Scattering of TeV-PeV cosmic ray particles with the turbulent interstellar magnetic field within the mean free path (i.e. a few 10s pc) can generate intermediate and small scale perturbations over an underlying large scale anisotropy (Giacinti and Sigl, 2011). The observed anisotropy structure, therefore, could be used to infer turbulence properties of the LIMF. At energies below about $100 \mathrm{TeV}$ the proton gyro-radius is a few thousands $\mathrm{AU}$, thought to be comparable to the length of the heliotail (Izmodenov and Alexashov, 2003). At these energies cosmic ray anisotropy is likely influenced by the extended and turbulent heliospheric magnetic field, and localized features in arrival direction can arise from the scattering of energetic cosmic ray particles with the heliospheric magnetic field ordered by the LIMF direction (Desiati and Lazarian, 2011).

Another model aimed to explain the origin of the TeV small scale anisotropic features, appeals to the observation that the two localized excess regions in the Morthern Hemisphere are seemingly close to the so-called Hydrogen Deflection Plane (HDP), which is the plane containing the directions of the interstellar flow and of the magnetic field upstream the heliospheric nose (Amenomori et al., 2011b). According to this model, cosmic rays propagating along the heliotail within the HDP are bent by the heliospheric magnetic field, so that two localized excess regions are formed symmetrically separated with respect to the direction of the heliotail on the HDP. This implies that the heliospheric magnetic field between about $70 \mathrm{AU}$ and $340 \mathrm{AU}$ along the heliotail is responsible for the two localized regions observed in the Northern Hemisphere in the energy range between 4 and $30 \mathrm{TeV}$. The heliospheric magnetic field has a complex structure determined by the combined effects of the 26 day rotation period of the Sun and of the $11 \mathrm{yr}$ solar cycle (Pogorelov et al., 2009a). This complex time-dependent magnetic field structure should produce an observable time variability in the relative intensity and position of the localized fractional regions over an $11 \mathrm{yr}$ period.

Some other models rely on an astrophysical origin of the observation. In Salvati and Sacco (2008); Drury and Aharonian (2008); Salvati (2010) it is noted that the two observed localized excess regions in the Northern Hemisphere, surround the present day apparent location of Geminga pulsar. The supernova that gave birth to the pulsar exploded about $340000 \mathrm{yr}$ ago, and the accelerated cosmic rays might have propagated along interstellar magnetic fields connecting the region of Geminga to Earth. Since nothing or very little is known of the local interstellar medium properties, cosmic ray diffusion is not sufficiently constrained to provide a coherent scenario that can explain the observations without considerable fine tuning.

Due to the coincidence of the most significant localized excess observed by Milagro with the heliotail, it is possible that we are seeing the effects of neutron production in the gravitationally focussed tail of the interstellar material, as suggested by Drury and Aharonian (2008). Cosmic rays propagating through the direction of the tail interact with matter and magnetic fields to produce neutrons and hence a localized excess of cosmic ray in that direction. But while the target size has about the right size compared to the decay length of multi-TeV neutrons $(\sim 0.1 \mathrm{pc})$, the increase of the gravitating matter density is too low to account for the observed excess.

In Malkov et al. (2010) it is proposed that cosmic rays emitted by a source (like a supernova remnant for instance) within a few $100 \mathrm{pc}$ are scattered by a strongly anisotropic Alfvén wave spectrum, formed by the turbulent cascade across the local field direction. Cosmic rays with small pitch angle with respect to the local interstellar magnetic field undergo the highest scattering, thus producing a faint localized excess region. An outer scale of the interstellar medium turbulence of about $1 \mathrm{pc}$ would explain the observations.

The fractional excess relative to the cosmic ray background observed by Milagro in the direction of the heliotail is $\sim 6 \times 10^{-4}$, i.e. about $1 / 10$ the amplitude of the global anisotropy at $\mathrm{TeV}$ energy. This is comparable to the amplitude that the broad tail-in excess would have if extrapolated 
from 100s $\mathrm{GeV}$ to $\mathrm{TeV}$ energies (Nagashima et al., 1998). Such an excess was found to be consistent with hadronic cosmic rays with an energy spectrum of the form $N(E) \sim$ $E^{-\gamma} e^{-E / E_{\mathrm{c}}}$ with spectral index $\gamma<2.7$ (i.e. flatter than the average cosmic ray spectrum) at $4.6 \sigma$ level, and a cut-off energy $E_{\mathrm{c}}=3-25 \mathrm{TeV}$ (Abdo et al., 2008). A similar spectral hardening was observed by ARGO-YBJ (Di Sciascio and the ARGO-YBJ Collaboration, 2012).

In this paper we discuss the scenario where the excess region of cosmic rays from the direction of the heliotail observed from a few tens $\mathrm{GeV}$ to about $10 \mathrm{TeV}$ is generated by re-acceleration processes of a fraction of energetic particles propagating through magnetic reconnection regions along the heliotail. A concurrent contribution from scattering with the turbulence ripples of the heliospheric magnetic field cannot be excluded, especially in relation to the other observed localized fractional excess regions, although this possibility is the topic of another paper (Desiati and Lazarian, 2011).

\section{Magnetic field structure at the heliotail}

The motion of the solar system through the local partially ionized medium ${ }^{1}$ produces a comet-like interface due to the solar wind plasma advected downstream by the interstellar flow, called the heliosphere. A termination shock, where the solar wind pressure equals that from the interstellar flow, is formed at approximately $100 \mathrm{AU}$ from the Sun. The interface separating interplanetary and interstellar magnetic fields, called heliopause, is at a distance of approximately $200 \mathrm{AU}$ in the upstream direction, and it may extend downstream several thousands AU (Izmodenov and Kallenbach, 2006) where it could be about $600 \mathrm{AU}$ wide (Pogorelov et al., 2009a). The LIMF drapes around the heliosphere, imprinting a deformation that affects its internal structure as well (Pogorelov et al., 2009b). The heliospheric magnetic field has been studied with detailed MHD simulations, where the effects from the 26 day solar rotation and the $11 \mathrm{yr}$ solar cycle were considered (Pogorelov et al., 2009a) (see also Scherer and Fahr, 2003). Over solar cycles the magnetic field polarity is reversed every $11 \mathrm{yr}$, generating unipolar regions dragged along the heliotail by the $\sim 100 \mathrm{~km} \mathrm{~s}^{-1}$ solar wind (Parker, 1979). In particular these magnetic regions grow to their maximum latitudinal extent during solar minimum (about 200-300 AU in size) and reduce to zero at solar maximum, when the heliospheric plasma is dominated by the strongly mixed polarity domains (about $0.1-1$ AU in size) from solar rotation (Nerney et al., 1995). Due to the tilt of the solar magnetic axis with respect to its rotation axis, the unipolar regions are thinner at lower latitudes (as shown in Fig. 2). Therefore, the tailward line of view is dominated

\footnotetext{
${ }^{1}$ the solar system is located at the edge of the so-called local interstellar cloud, which is part of a complex cloudlet structure expanding from the Scorpion-Centaurus Association (see Frisch et al., 2011)
}

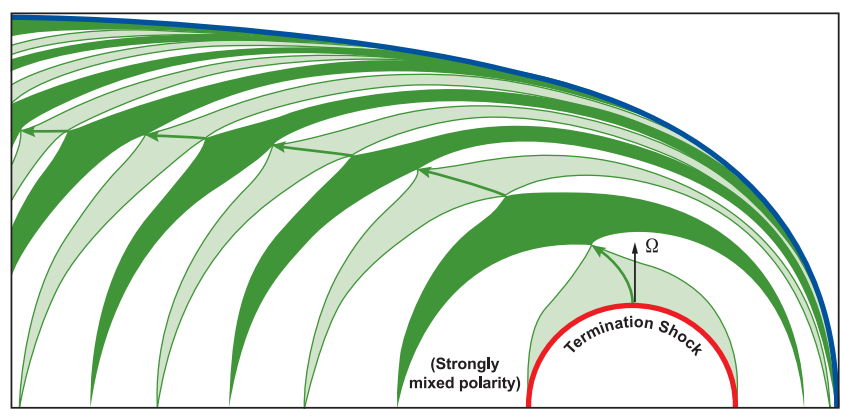

Fig. 2. A schematic meridional view of the sectored heliospheric magnetic field arising from the 11-yr solar cycle polarity reversals. The subsonic solar wind pushes the sectors across the termination shock along the heliotail, compressing them to each other and toward the heliopause. The turbulence that is expected to perturb the heliospheric magnetized plasma, determines the thickness of the current sheet outflow regions. While their length depends on the average magnetic field geometry and by the turbulence level. Adapted from Nerney et al. (1995); Lazarian and Opher (2009).

by the finely alternating magnetic field, while along sighlines away from it the magnetic domains have larger size. MHD numerical simulations show that the sectored unipolar magnetic field regions can propagate for several solar cycles before they dissipate into the local interstellar medium. The corresponding periodic variations on the heliospheric plasma induce changes in the magnitude of the Alfvén velocity by about $20 \%$, and of the magnetic field by about $25 \%$ (Pogorelov et al., 2009a).

There is observational evidence that the plasma in the heliosheath has Reynolds number $R_{e} \approx 10^{14}$ (see Lazarian and Opher, 2009 and references therein), meaning that the strength of non-linear convective processes at the largest scale is more important than the damping viscous processes in the dynamics of the flow. We expect a similarly high Reynolds number in the inner heliotail as well. In such conditions it is very unlikely that plasma flow stays laminar, and the downstream motion in the heliotail is likely turbulent. In addition, the presence of neutral atoms in the partially ionized local cloud medium (where the solar system is moving) is essential for the dynamics of the heliosphere and LIMF interaction. Charge-exchange processes between the interstellar inflowing neutral atoms and the outflowing solar wind protons can produce Rayleigh-Taylor type instabilities on the heliopause with amplitude of a few tens AU and over a time scale of a few hundreds years (Liewer et al., 1996). Also in a model of plasma-neutral fluid coupled via collision and charge-exchange processes, it is found that such non-linear coupling leads to alternate growing and damping of Alfvénic, fast and slow turbulence modes, at $L \sim 100$ s AU scale and with evolution time longer than inertial time $L / V_{\mathrm{A}}$ (Shaikh and Zank, 2010), with $V_{\mathrm{A}}$ the Alfvén velocity. Such modulations can propagate on the heliopause, producing ripples along the heliotail that can penetrate deep inside the 
heliosheath and propagate outward into the local interstellar medium. Therefore, although more investigations are needed in order to understand the detailed plasma properties in the heliotail and its outer boundary, it is reasonable to assume here that magnetic fields in the heliotail are weakly stochastic, and likely reconnecting as the gradient in solar wind advects magnetic field lines closer to each other. The Alfvén velocity of the turbulence in the heliotail is expected to be approximately $40-70 \mathrm{~km} \mathrm{~s}^{-1}$, with the actual value depending on the location within the sectored magnetic field (Pogorelov et al., 2009a). This is smaller than the solar wind speed downstream the termination shock, therefore magnetic reconnection in the heliotail is not expected to change the overall magnetic field structure. Nevertheless, the effects of turbulence are very important from the point of view of magnetic reconnection and the particle acceleration that it entails.

Simulations of the magnetic fields in the heliotail are extremely challenging due to its extension and to the complex interaction with the interstellar wind and between heliospheric magnetic field and the LIMF, but mainly because there is currently no direct data collection from this remote portion of the heliosphere. Future refinements of MHD simulations will provide higher resolution mapping of the heliotail and of the plasma properties, that will help improving our knowledge of its effects on $\mathrm{TeV}$ cosmic ray propagation.

\section{Stochastic magnetic reconnection}

Astrophysical plasmas are often highly ionized and magnetized (Parker, 1970), and they undergo dissipative processes, which annihilate the magnetic fields and convert electromagnetic energy into plasma energy. Due to these processes, plasma from regions of a given polarity becomes magnetically connected to that of opposite polarity: this is when magnetic reconnection occurs. However, reconnection speed, and therefore the rate at which magnetic energy is converted into plasma energy, is too small to be important for acceleration of energetic particles, unless the effects of plasma resistivity are negligible.

In the Sweet-Parker model of reconnection (Sweet, 1958; Parker, 1957) the outflow is limited within the transition zone $\Delta$, which is determined by Ohmic diffusivity (see top of Fig. 3). In this model reconnection speed is smaller than the Alfvén velocity of the plasma by a factor equal to $S^{-1 / 2}=\left(L V_{\mathrm{A}} / \eta\right)^{-1 / 2}$, where $S$ is the Lundquist number, $L$ the length of the current sheet, $V_{\mathrm{A}}$ the Alfvén speed and $\eta$ is the Ohmic resistivity of the plasma. The length of the current sheet is determined by the extent of magnetic flux tubes that get in contact. Although the properties and dimensions of the heliotail are not well constraint yet, it is possible to state that the extension of current sheets between sectored heliospheric magnetic field in the heliotail could lay between about $100 \mathrm{AU}$ and $300 \mathrm{AU}$ (Pogorelov et al., 2009a). Assuming the same plasma properties as in the heliosheath
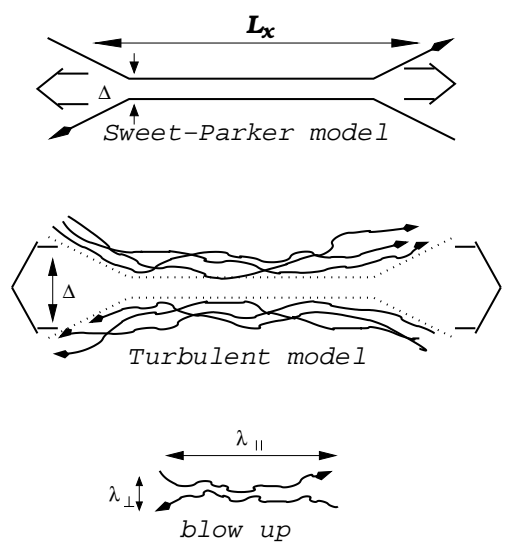

Fig. 3. Upper plot: Sweet-Parker model of reconnection (Sweet, 1958; Parker, 1957). The outflow region is limited within a thin transition zone $\Delta$ between the reversed magnetic field lines, which depends on plasma resistivity. The other scale is an astrophysical scale $L \gg \Delta$. Middle plot: reconnection of weakly stochastic magnetic field according to Lazarian and Vishniac (1999). The outflow region is determined by the diffusion of magnetic field lines, which depends on the field stochasticity. Lower plot: an individual small scale reconnection region. The reconnection over small patches of magnetic field determines the local reconnection speed. The global reconnection speed is substantially larger as many independent patches come together. The bottleneck for the process is given by magnetic field wandering and it becomes comparable to $L$ as the turbulence injection velocity approaches the Alfvenic one. From Lazarian et al. (2004).

closer to the heliospheric nose, $S$ is about $10^{12}-10^{13}$ (Lazarian and Opher, 2009). Therefore, the corresponding reconnection speed for the Sweet-Parker model is several orders of magnitude smaller than the Alfvén velocity. In fact in this case plasma collected over the size $L$ should be ejected with speed $\sim V_{\mathrm{A}}$ through the outflow region of thickness $\Delta=L S^{-1 / 2}$, i.e. much smaller than the length of the current sheet. It is the large difference between $L$ and $\Delta$ that makes reconnection slow and unlikely to produce any effect on the plasma. The major consequence of such a model is that reconnection speed is limited by Ohmic resistivity of the plasma (see right panel of Fig. 4). Since most astrophysical plasmas have very low resistivity, a magnetic reconnection mechanism such this would not produce observable effects.

On the other hand, various observations suggest that reconnection, when it occurs, can be fast in some circumstances. For instance, the development of solar flares suggests that magnetic reconnection should be initially slow in order to ensure the accumulation of magnetic flux, and then suddenly becomes fast in order to explain the observed fast release of energy. Fast reconnection would require $L \sim \Delta$, meaning that the region over which magnetic flux tubes intersect is comparable to the size of the outflow region. This can be achieved by increasing the outflow region beyond the prediction of Sweet-Parker model, or by making $L$ as 

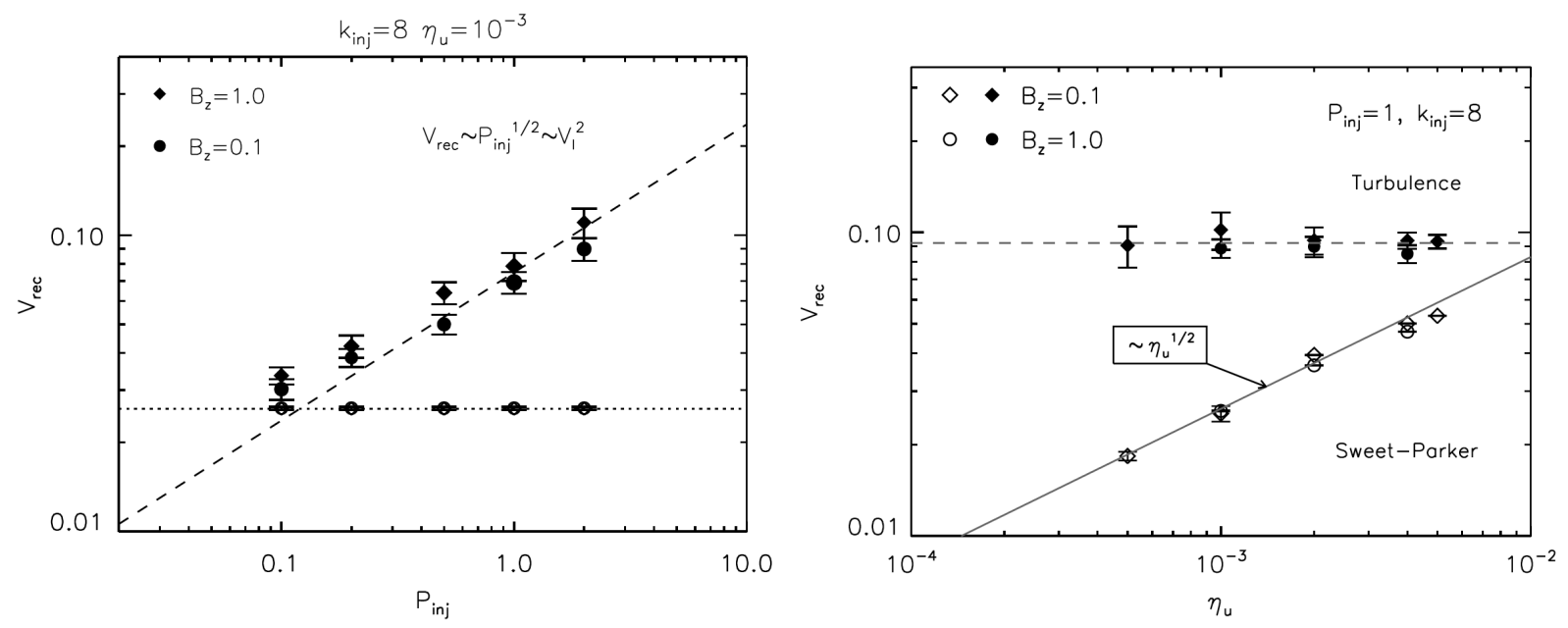

Fig. 4. Left panel: dependence of the reconnection speed $V_{\mathrm{R}}$ on injection power $P_{\text {inj. }}$ Right panel: dependence of the reconnection speed $V_{\mathrm{R}}$ on the uniform resistivity $\eta_{\mathrm{u}}$. Open symbols are for Sweet-Parker reconnection scenario (Sweet, 1958; Parker, 1957), and filled symbols are for weakly stochastic reconnection scenario (Lazarian and Vishniac, 1999). See Kowal et al. (2009); Lazarian et al. (2011).

small as the Ohmic diffusion region, so that magnetic field lines reconnect in an "X-point". In this X-point collisionless model (Petschek, 1964), reconnection speed does not depend on the resistivity and it is of the order of Alfvén velocity of the plasma. On the other hand, X-points are found to be unstable and to collapse into a Sweet-Parker current sheet in the MHD regime (Biskamp, 1996). In a collisionless plasma X-point, stability can be maintained through coupling to a dispersive plasma mode (Sturrock, 1966; Shay and Drake, 1998). Recently it was discovered that X-points can be stabilized in the presence of MHD Hall effect so that the outflow opens up on larger scale, thus making reconnection fast (Shay et al., 1998, 2004). On the other hand, most astrophysical plasmas are turbulent, and the heliosphere is most probably not an exception. This means that X-points can be created by turbulence and sustained at small scale by HallMHD effects, but only until turbulence itself collapses the $\mathrm{X}$-points to form extended thick outflow regions (as observed by Ciaravella and Raymond (2008) in multi-frequency observations of solar flares). Even without turbulence it has been suggested that magnetic islands dynamically produced at Xpoints tend to be volume filling and to produce thick collisionless reconnection regions (Drake et al., 2006), with high reconnection speed. However, since turbulence is likely ubiquitous in astrophysics plasmas, we concentrate here on fast reconnection mechanisms in weakly stochastic plasmas.

A model of fast magnetic reconnection that generalizes the Sweet-Parker scheme for the case of weakly stochastic magnetic fields was proposed by Lazarian and Vishniac (1999) (henceforth LV99). Even though the notion of reconnection affected by turbulence is not new, in the LV99 model it is recognized that turbulence can decouple the width of plasma outflow region from the scale determined by Ohmic effects. In fact the outflow width is limited by the diffusion of magnetic field lines, which depends on turbulence only (see center of Fig. 3), and can be much wider than the thickness of the individual current sheets (see bottom of Fig. 3). Although reconnection events happen on small scales $\lambda_{\|}$, where magnetic field lines get into contact, a number of independent reconnection processes takes place simultaneously over extended macroscopic current sheets $L \gg \lambda_{\|}$within a wide outflow region $\Delta \sim L$. Therefore, the effective reconnection rate is not limited by the speed of individual Sweet-Parker events on scale $\lambda_{\|}$(where plasma resistivity plays a dominant role), instead, it is enhanced by the large $\Delta$ that depends on the magnetic field wandering. In such a situation it was found that reconnection speed is close to the turbulent velocity in the plasma. In particular, assuming isotropically driven turbulence characterized by an injection scale $l \lesssim L$ the reconnection speed is (Lazarian and Vishniac, 1999; Lazarian, 2006)

$V_{\mathrm{R}} \approx V_{\mathrm{A}}\left(\frac{l}{L}\right)^{1 / 2}\left(\frac{V_{1}}{V_{\mathrm{A}}}\right)^{2}$

where $V_{1}$ is the turbulent velocity at the largest scale and $V_{\mathrm{A}}$ the Alfvén velocity. Since turbulence in the heliotail is assumed to be weak, magnetic perturbations are comparably smaller with respect to the mean heliospheric magnetic field, therefore $V_{1} \lesssim V_{\mathrm{A}}$ (i.e. turbulence in the heliotail is subAlfvénic).

Numerical MHD calculations of weakly stochastic magnetic reconnection were performed by Kowal et al. (2009) and they proved that reconnection is fast and independent of Ohmic resistivity of the plasma, as shown in right panel of Fig. 4, while it depends on the power of injected turbulence (shown in left panel of Fig. 4). In these simulations, turbulence is preexisting and not related to reconnection processes. By varying the Ohmic and anomalous resistivity of 
the plasma, reconnection rate is not affected, confirming that in the presence of turbulence resistivity is not important.

In the LV99 model, reconnection develops while the wide outflow region is filled with turbulent reconnected magnetic field lines moving in opposite directions. In fact, numerical simulations by Kowal et al. (2011) show that the interface between the oppositely oriented magnetic fields has a much more complex topology if compared to the Sweet-Parker reconnection mechanism, and also to the schematic representation in Fig. 3 (see Sect. 5). The outflow volume is filled with enhanced current density regions with loops of reconnected magnetic flux, where locally reconnection works faster since the current density reaches higher values. The magnetic loops shrink as a dynamical consequence of reconnection development, while multiple reconnection events happen at the same time due to the magnetic field stochasticity.

\section{Acceleration in reconnection regions}

Electric fields associated with reconnection events can accelerate energetic particles. For a particle of charge $q$, the typical energy gained in a reconnection process is of the or$\operatorname{der} q\left(V_{\mathrm{R}} / c\right) B \lambda_{q}$, where $\lambda_{q}$ is the coherence length of the particle within the reconnection layer. Efficient acceleration would require, therefore, both $V_{\mathrm{R}}$ and $\lambda_{q}$ to be large. However, in general in any fast reconnection mechanism, the fraction of volume that is subject to resistive effects and reveals strong electric fields is small and most of the magnetic energy is converted into kinetic energy of the plasma instead. Therefore, only a small fraction of the energy can be transferred through any fast reconnection process to energetic particles if a direct electric field is involved. The observation of a large normal component of the electric field near an X reconnection point in the Earth's magnetotail was interpreted as Hall electric field at the X-point current sheet by Wygant et al. (2005), capable of accelerating ions to $\sim 10 \mathrm{~s} \mathrm{keV} \mathrm{scale.}$ In this paper we concentrate on the mechanism of energetic particle re-acceleration in weakly stochastic reconnection regions.

In the LV99 mechanism, reconnection speed can approach $V_{\mathrm{A}}$, which can be appreciably large, therefore particles entrained on reconnecting field lines bounce back and forth between the approaching magnetic walls while staying on the field lines that are contracting. This results in an increase of particle velocity with every bouncing, as discussed by de Gouveia Dal Pino and Lazarian $(2003,2005)$ (see also Lazarian, 2005), where it was shown that reconnection induces particle acceleration. The effect that individual magnetic loops shrinking in the reconnection region have on energetic particles is equivalent to that of first order Fermi acceleration in magnetic mirrors. Figure 5 schematically represents the simplest realization of acceleration within the reconnection region expected within LV99 model. As energetic particles bounce back and forth between converging

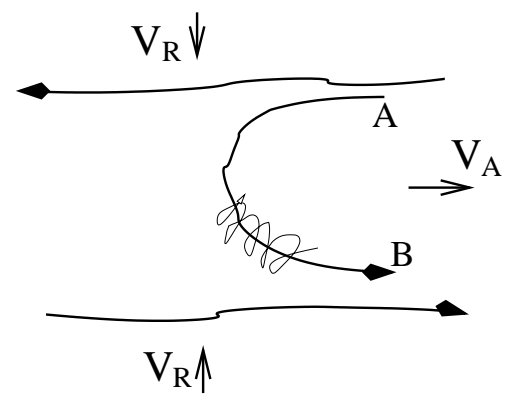

Fig. 5. The simplest realization of magnetic reconnection at small scale and of acceleration as an energetic particle bounces back and forth between converging magnetic field lines. The converging velocity determines the reconnection speed $V_{\mathrm{R}}$, while the advection of the accelerated particles entrained on the magnetic field lines, occurs at an outflow speed that in most cases is of order the Alfvén velocity of the plasma $V_{\mathrm{A}}$. Particles bouncing at points $\mathrm{A}$ and $\mathrm{B}$ happens because either of streaming instability induced by energetic particles or magnetic turbulence in the reconnection region. In an actual turbulent plasma, the outflow region, at large scale, is filled with reconnecting loops and current sheets, each of which a possible acceleration site (see text). From Lazarian (2005).

magnetic fluxes, they gain energy. In the figure, the bouncing at points A and B is just an illustration of the process. In reality particles never pass by the same points in 3-D, but they locally stream along magnetic field lines and bounce back and forth through magnetic bottles that form in the reconnection region.

The simple acceleration process represented in Fig. 5 can be easily quantified. An energetic particle with energy $E$ bouncing back and forth between a magnetic mirror will gain an energy $\Delta E \sim\left(V_{\mathrm{R}} / c\right) E$ in every cycle. The process continues until particles gain enough high energy to either diffuse perpendicularly out of the reconnection region or get ejected by the outflow plasma at the Alfvén velocity. This last possibility was considered by de Gouveia Dal Pino and Lazarian $(2003,2005)$, namely that particle diffusion perpendicular to the mean magnetic field is negligible. Perpendicular diffusion arises from magnetic field wandering as particles scatter marginally perpendicular to the local magnetic field. This effect was accounted by Yan and Lazarian (2004, 2008 ) to describe cosmic ray propagation, for instance. As mentioned, the properties of turbulent plasma in the heliotail are not well know at this point, therefore it is difficult to quantify the diffusion regime; nevertheless, in general perpendicular scattering in sub-Alfvénic turbulence is found to be subdominant with respect to parallel scattering (Yan and Lazarian, 2008; Beresnyak et al., 2011) (see also Lazarian, 2006, 2007). In this case, the energy spectrum of accelerated test particles ${ }^{2}$ is (de Gouveia Dal Pino and Lazarian, 2003; Lazarian and Opher, 2009)

\footnotetext{
2i.e. neglecting the back-reaction of accelerated particles, see Longair (1992)
} 
$N(E) d E \sim E^{-5 / 2} d E$.

If perpendicular diffusion in the reconnection region is not negligible, particles bouncing between approaching reconnecting field lines of the magnetic mirror are not confined as within walls, but can keep bouncing while reconnection proceeds. In this situation particles may have cross field propagation but cannot escape from the large scale reconnection region, producing a spectrum asymptotically reaching $N(E) d E \sim E^{-1} d E$.

In case of re-acceleration of cosmic rays with a seed spectrum $E^{-2.7}$, after acceleration it still becomes $\propto E^{-5 / 2}$, i.e. harder than the initial spectrum. It is important to note that the expected energy spectrum in Eq. (2) is an estimate based on a rather idealized situation. The derivation above considers only particles bouncing back and forth between the two reconnection layers. The actual picture of stochastic reconnection in the LV99 model includes many simultaneous reconnection events happening at different scales throughout the reconnection volume. Figure 6 (from Kowal et al., 2011) shows an evolved 2-D snapshot of magnetic field configuration during reconnection from a nearly incompressible nonresistive MHD domain simulation without including kinetic effects, such as pressure anisotropy, the Hall term, or anomalous effects. The initial condition of the domain was set with eight Harris current sheets in a periodic box and a density profile corresponding to a uniform total pressure. A perturbation with random weak velocity fluctuations was used to enable spontaneous reconnection events. It is evident from the figure that the reconnection volume is filled with magnetic loops (or islands) and that several reconnection events occur at the same time within the loops and along the current sheets between the loops. The simulation shows the existence of merging loops with their resulting deformation and contraction that provide appropriate conditions for particle acceleration. This picture is very similar to the 2-D simulation by Drake et al. (2010), where islands, or loops, are in fact only the 2-D projections of 3-D magnetic tubes, as shown in Kowal et al. (2011)

The topological complexity of the reconnection region may have an influence on the actual spectral shape of the accelerated particles. Each local reconnection region (within a magnetic loop or a current sheet) provides the accelerated spectrum $\propto E^{-5 / 2}$. But when energetic particles cross several reconnection regions they undergo further acceleration with a seed spectrum, corresponding to that gained within the previously crossed reconnection region. It is well known from the theory of diffusive shock acceleration that the supply of a seed power law spectrum into an acceleration region leads to an amplification of the distribution without changing the spectral index. However the increased number of high energy particles is accompanied by a decrease of the number density at the low energy cutoff, leading to a flattening of the distribution at intermediate energy (see Bell, 1978; Melrose

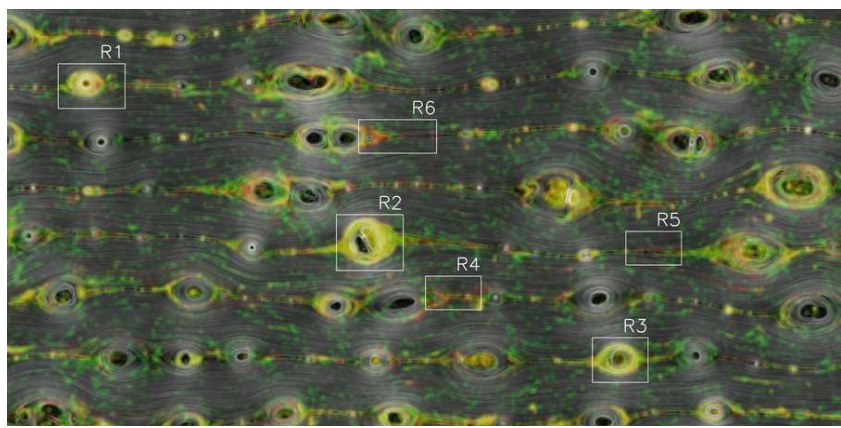

Fig. 6. Evolved 2-D snapshot of magnetic field configuration where eight parallel Harris current sheets were perturbed in order to trigger plasma instabilities and reconnection events (represented in grey shades). 10000 test particles, with initial thermal distribution with temperature corresponding to the sound speed of the MHD model were injected in this plasma snapshot to study the acceleration mechanism induced by magnetic reconnection. The red and green colors correspond to regions where either parallel or perpendicular acceleration occurs, respectively, while the yellow color shows locations where both types of acceleration occur. The parallel component increases in the contracting islands and in the current sheets as well, while the perpendicular component increases mostly in the regions between current sheets. The white boxes correspond to the sites where detailed determination of acceleration properties were done (see text). From Kowal et al. (2011).

and Pope, 1993; Gieseler and Jones, 2000), and, therefore, to a harder energy spectrum. The exact slope of the final spectrum after bouncing within and escaping from several reconnection regions depends on the cutoff energy, and on energy loss processes that particles undergo during acceleration and, most importantly, between acceleration processes. Scattering, for instance can degrade particle energy so that at each acceleration step a new low energy population is seeded into the process, leading to a softening of the spectrum.

Non-linear effects from back-reaction of the accelerated particles might be important in reconnection processes. However, so far the only approach to address back-reaction of particles on reconnecting plasma involved electrons (Drake et al., 2006). In fact the evidence of back-reaction can be found in the simulations of test particles propagating in the magnetotail (Birn et al., 2004) and also in test particles studies in MHD models with magnetic islands (Matthaeus et al., 1984; Kliem, 1994). In Drake et al. (2006), where acceleration occurs in contracting loops formed in 2-D collisionless reconnection, back-reaction is introduced by the term $\left(1-8 \pi \bar{\epsilon}_{\|} / B^{2}\right)$, where $\overline{\epsilon_{\|}}$is the energetic particle parallel energy averaged over the distribution of particle velocities. This would produce an accelerated spectrum $\sim E^{-3 / 2}$, instead of the steeper $\sim E^{-5 / 2}$.

Numerical simulations of test particles injected in the domain represented in Fig. 6, with initial thermal distribution, show that particle velocity parallel (in red) and perpendicular (in green) to the mean magnetic field increases. Yellow 

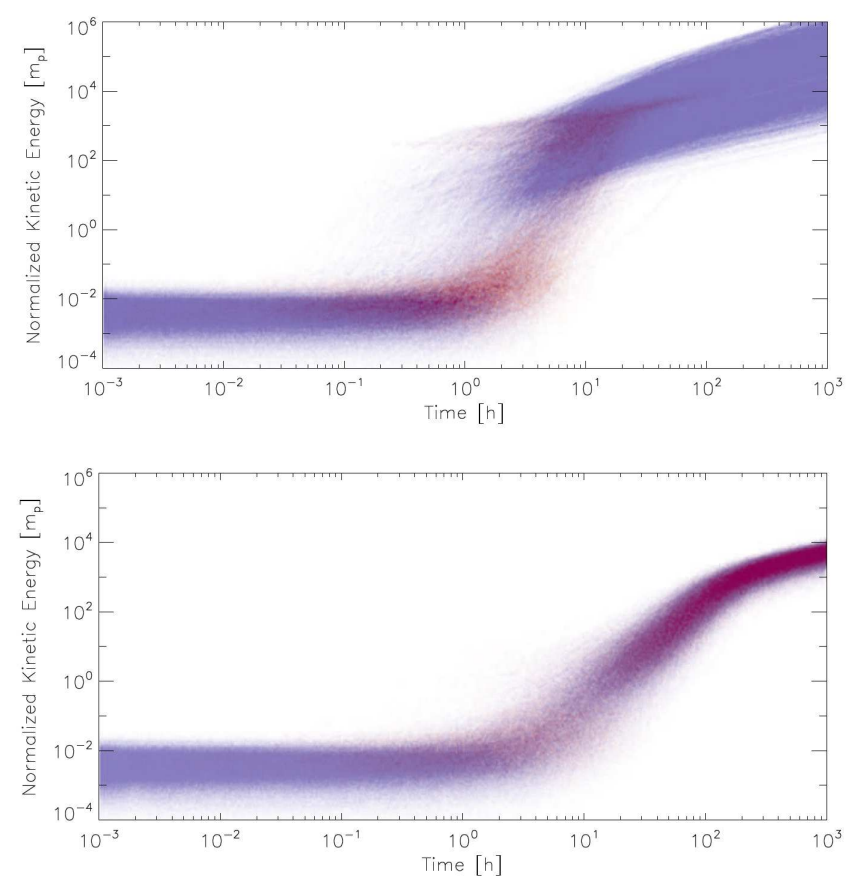

Fig. 7. Top panel: kinetic energy evolution in time of 10000 protons in a 2-D model of reconnection (i.e. with magnetic field on a plane). Bottom panel: the same distribution but in a fully 3-D model of reconnection. In 2-D the perpendicular component of particle velocities (in blue) becomes dominant over time with respect to the parallel component (in red). While in a 3-D domain it is the parallel component to dominate. The energy is normalized to the proton rest mass. The background magnetized flow with multiple current sheet layers is at time 4.0 in Alfvén time units in the model. Note that the transition from exponential energy grow to nearly linear occurs when the largest loop in the plasma reaches the size of a few tens the size of simulation box. From Kowal et al. (2011).

color indicates the locations where acceleration increases both components without preference. But while in 2-D domains perpendicular velocity mostly increases over longer integration time, in 3-D, where the loops develop in magnetic tubes, there is no such a limitation making acceleration in the parallel dimension dominant (Kowal et al., 2011, 2012) as shown in Fig. 7, where velocities were sampled within the regions indicated with white boxes in Fig. 6. It is found that within contracting/deforming magnetic loops or current sheets, particles accelerate mainly through first order Fermi acceleration with particles bouncing back and forth between converging mirrors (de Gouveia Dal Pino and Lazarian, 2003, 2005; Drake et al., 2010), while outside these regions particles mostly undergo drift acceleration from magnetic field gradients. It is possible that turbulence far from loops, current sheets and diffusion regions favors mostly second order Fermi acceleration mechanisms with particles being scattered by approaching and receding magnetic irregularities. In Kowal et al. (2012) it was argued that second order Fermi acceleration is the dominant process in purely turbulent plasmas with no converging flow, although its rate is reduced. Moreover, reconnection layers in pure turbulence could be responsible for first-order Fermi acceleration of low energy particles. However, more studies are needed to fully understand the interplay between different acceleration mechanisms in turbulent media.

In shock acceleration, particles gain energy via plasma compression differences between the upstream and downstream regions. Energy gain is described by Parker's transport equation (Parker, 1965), which was derived in the limit of strong scattering, and it is explicitly driven by plasma compression. On the other hand in magnetic reconnection it is possible to have acceleration even in an incompressible plasma. In fact numerical MHD simulations such as the one by Kowal et al. $(2009,2011,2012)$ were done in a nearly incompressible regime, and as long as there is no strong scattering to maintain plasma isotropy, the parallel energy gain dominates and, as a result, the particles entrained in the reconnecting magnetic flux gain net energy.

\section{Re-acceleration of cosmic rays}

The sectored heliospheric magnetic field regions in the heliotail generated by the $11 \mathrm{yr}$ solar cycle is composed of 100-300 AU wide unipolar domains with turbulence scale likely of order $10-100 \mathrm{AU}$, although the injection scale is not known precisely. The downstream solar wind motion in the heliotail induces converging flows in the turbulent plasma that ignite reconnection. As discussed in Sect. 4, turbulence creates the conditions for forming multiple simultaneously reconnecting magnetic fluxes (loops and current sheets) throughout large portions of the plasma. Acceleration takes place across the entire reconnection region and energetic particles are accelerated through a sequence of multiple reconnection events (see Sect. 5). Although second order Fermi acceleration from pure turbulence may occur as well, as long as reconnection is efficient, first order Fermi acceleration is dominant. The overall process, therefore, takes place across regions that are comparable with the size of the unipolar magnetic domains, or even the size of the heliotail itself. The energy spectrum of those re-accelerated particles is, in the simplest case, $\sim E^{-\gamma}$, with spectral index $\gamma=5 / 2$ or smaller as discussed in Sect. 5, which is flatter than the mean cosmic ray spectrum. Such acceleration can occur for as long as the cosmic ray particles are trapped within the reconnection regions. Using the simple argument that the gyroradius should not be larger than the size of the magnetized region $L_{\text {zone }}$, the maximum energy for a proton is (Longair, 1992)

$E_{\max } \approx 0.5\left(\frac{B}{1 \mu \mathrm{G}}\right)\left(\frac{L_{\text {zone }}}{100 \mathrm{AU}}\right) \mathrm{TeV}$.

The magnetic field strength in the heliotail is not known with precision, but we can assume that it is of the order of $1-4 \mu \mathrm{G}$ (Pogorelov et al., 2009a). $L_{\text {zone }}$ is assumed to 
be within the range 100-500 AU (the higher bound being approximately the heliotail thickness), therefore the maximum energy that cosmic rays can achieve is approximately between $0.5-10 \mathrm{TeV}$. This means that the fractional excess region observed in the direction of the heliotail is likely expected to have a harder spectrum than the average cosmic rays up to about $1-10 \mathrm{TeV}$. Above this energy the spectrum transitions back to the steeper $\sim E^{-2.7}$. Scattering processes within the heliotail can mitigate the acceleration effects and the related cosmic ray distribution at a given energy. Based on the global magnetic field structure in the heliotail, confirmed in MHD simulations (see Sect. 3), TeV cosmic particles experience the lowest scattering along the line of sights parallel to the interstellar downstream flow, where magnetic field is strongly mixed at small scale. While away from this direction scattering in the unipolar magnetic domains scrambles particles direction and effectively reduces the overall acceleration efficiency. At sub-GeV energies, the stronger scattering along the heliotail would degrade anisotropy and spectral features. As stated in Lazarian and Desiati (2010), the properties of magnetic reconnections are still under extensive study, and their level of complexity is being subject of debate. At the same time the very little explored tail region of the heliosphere, makes the problem under discussion here even more uncertain. However, it is suggestive that the observation of $\mathrm{TeV}$ cosmic ray arrival distribution and energy spectrum over small angular regions could be used to probe properties over the most remote regions of the heliosphere.

Although there is no energy spectral determination in the sub-TeV energy range, the significant hardening of the spectrum observed by Milagro (Abdo et al., 2008) and ARGOYBJ (Di Sciascio and the ARGO-YBJ Collaboration, 2012) is indicative of a possible re-acceleration mechanism that involves a fraction of cosmic rays propagating from the direction of the heliotail. While waiting for other experimental results that can confirm a harder than average energy spectrum of cosmic rays within the localized excess region, the energy flux corresponding to the $\sim 6 \times 10^{-4}$ fractional excess from $\sim 10 \mathrm{GeV}$ to a few tens of $\mathrm{TeV}$ can be estimated to be approximately between $10^{-9}$ and $10^{-8} \mathrm{erg} \mathrm{cm}^{-2} \mathrm{~s}^{-1}$, for $\gamma=2.7-2.0$, respectively. The corresponding average power dissipated in the re-acceleration of such energetic particles is approximately between $10^{20}$ and $10^{22} \mathrm{erg} \mathrm{s}^{-1}$. Even though a precise quantitative assessment of the power necessary to reaccelerate the fraction of energetic cosmic rays forming the Milagro localized excess region is not possible at this point, this simple estimation shows that the fraction of heliospheric plasma power dissipated into cosmic ray kinetic energy is very small if compared to that transported by the solar wind $\left(\approx 10^{27} \mathrm{erg} \mathrm{s}^{-1}\right.$, see Parker, 1962).

It is interesting to note that within the last few years, experimental evidence that cosmic ray spectrum becomes harder at about $0.2-0.3 \mathrm{TeV} /$ nucleon has been accumulated by ATIC-2 (Wefel et al., 2008), CREAM (Ahn et al., 2010) and PAMELA (Adriani et al., 2011). In particular the
CREAM results seem to suggest that cosmic ray spectrum may become softer again at about $10 \mathrm{TeV} /$ nucleon, although more observation is needed on this regard. The direct observation of a correlation between spectral features and arrival direction would provide a breakthrough on the role of the heliotail in the $\mathrm{TeV}$ cosmic ray properties.

\section{Conclusions}

The observation that cosmic rays are anisotropic has gained special attention in the last decade, since it could provide information about the galactic sources of the energetic particles and about the properties of the local interstellar medium and of the heliospheric magnetized plasma. Of particular interest is the evolution with energy of its angular structure, especially of the tail-in anisotropy which appears as a broad excess at sub-TeV energies from the direction of the heliotail, and seemingly degenerate into separate localized fractional excess regions above a few TeV. The directional coincidence of the tail-in excess at sub-TeV energies, and of the most significant of the localized fractional excess regions at $\mathrm{TeV}$ energies with the heliotail provides a compelling connection to this little known extended portion of the heliosphere.

Although we cannot exclude that other phenomena occur and might dominate the origin of the observation, such as the effect of energetic cosmic ray interaction with the turbulent ripples along the heliotail, in this paper another mechanism is discussed. Namely that a fraction of cosmic rays propagating through the heliotail are re-accelerated via first-order Fermi acceleration mechanism in weakly stochastic magnetic reconnection processes that originate in sectored magnetic field domains produced by the 11-yr solar cycle. In general, 3-D numerical simulation show that such an acceleration mechanism can be efficient up to a few $\mathrm{TeV}$, where a flatter than average spectrum could arise, depending on the competing effects of multiple acceleration processes and escape or loss effects, and back-reaction. On the other hand, the properties of magnetized plasma in the heliotail are not yet fully understood, therefore details of cosmic ray propagation in this region are still uncertain. Sub-TeV cosmic rays may be accelerated over extended regions and may undergo more scattering, thus producing a broader arrival distribution. While multi- $\mathrm{TeV}$ cosmic rays undergo more efficient acceleration and their localized substructure in arrival direction are more related to the acceleration sites along the heliotail, such acceleration mechanism is intrinsically anisotropic and as long as scattering is sub-dominant it would generate a net energy gain that could explain the seemingly harder spectrum observed within the localized excess regions by Milagro.

Acceleration processes in weakly stochastic magnetic reconnection regions as described by de Gouveia Dal Pino and Lazarian (2003) have been used in Lazarian and Opher (2009) to explain the origin of the anomalous cosmic rays. The Voyager spacecraft measurements show that the 
anomalous cosmic rays persist also downstream the termination shock, indicating that the site of their acceleration is within the heliosheath closer to the heliopause in the upstream interstellar flow direction. The sectored magnetic field arising from the 26 day solar rotation and originated by the difference between rotation and magnetic axes are pushed away by the solar wind and compressed upstream toward the heliopause, causing magnetic reconnection and energetic particle acceleration. A similar model for the origin of anomalous cosmic rays was proposed by Drake et al. (2010) where the process of collisionless reconnection was discussed. In this paper we discussed a similar mechanism of the re-acceleration of energetic cosmic ray particles, where the scale of the sectored magnetic field is significantly larger. The higher magnetic energy involved provides the possibility to accelerate higher energy particles in an observable manner in terms of a slightly anomalous energy spectrum and arrival distribution.

Acknowledgements. The authors would like to thank the reviewers who helped to improve the paper. A. L. acknowledges the support of the NSF grant AST 0808118, NASA grant X5166204101 and of the NSF-sponsored Center for Magnetic Self-Organization. P. D. acknowledges the support from the US National Science Foundation-Office of Polar Programs.

Edited by: G. Lapenta

Reviewed by: three anonymous referees

\section{References}

Abbasi, R., Abdou, Y., Abu-Zayyad, T., Adams, J., Aguilar, J. A., Ahlers, M., Andeen, K., Auffenberg, J., Bai, X., Baker, M., and et al.: Measurement of the Anisotropy of Cosmic-ray Arrival Directions with IceCube, Astrophys. J. Lett., 718, L194-L198, doi:10.1088/2041-8205/718/2/L194, 2010.

Abbasi, R., Abdou, Y., Abu-Zayyad, T., Adams, J., Aguilar, J. A., Ahlers, M., Altmann, D., Andeen, K., Auffenberg, J., Bai, X., and et al.: Observation of Anisotropy in the Arrival Directions of Galactic Cosmic Rays at Multiple Angular Scales with IceCube, Astrophys. J., 740, 16, doi:10.1088/0004-637X/740/1/16, 2011.

Abbasi, R., Abdou, Y., Abu-Zayyad, T., Ackermann, M., Adams, J., Aguilar, J. A., Ahlers, M., Allen, M. M., Altmann, D., and et al.: Observation of an Anisotropy in the Galactic Cosmic Ray arrival direction at $400 \mathrm{TeV}$ with IceCube, Astrophys. J., 746, 33, doi:10.1088/0004-637X/746/1/33, 2012.

Abdo, A. A., Allen, B., Aune, T., Berley, D., Blaufuss, E., Casanova, S., Chen, C., Dingus, B. L., Ellsworth, R. W., Fleysher, L., Fleysher, R., Gonzalez, M. M., Goodman, J. A., Hoffman, C. M., Hüntemeyer, P. H., Kolterman, B. E., Lansdell, C. P., Linnemann, J. T., McEnery, J. E., Mincer, A. I., Nemethy, P., Noyes, D., Pretz, J., Ryan, J. M., Parkinson, P. M. S., Shoup, A., Sinnis, G., Smith, A. J., Sullivan, G. W., Vasileiou, V., Walker, G. P., Williams, D. A., and Yodh, G. B.: Discovery of Localized Regions of Excess 10-TeV Cosmic Rays, Phys. Rev. Lett., 101, 221101, doi:10.1103/PhysRevLett.101.221101, 2008.
Abdo, A. A., Allen, B. T., Aune, T., Berley, D., Casanova, S., Chen, C., Dingus, B. L., Ellsworth, R. W., Fleysher, L., Fleysher, R., Gonzalez, M. M., Goodman, J. A., Hoffman, C. M., Hopper, B., Hüntemeyer, P. H., Kolterman, B. E., Lansdell, C. P., Linnemann, J. T., McEnery, J. E., Mincer, A. I., Nemethy, P., Noyes, D., Pretz, J., Ryan, J. M., Parkinson, P. M. S., Shoup, A., Sinnis, G., Smith, A. J., Sullivan, G. W., Vasileiou, V., Walker, G. P., Williams, D. A., and Yodh, G. B.: The Large-Scale Cosmic-Ray Anisotropy as Observed with Milagro, Astrophys. J., 698, 21212130, doi:10.1088/0004-637X/698/2/2121, 2009.

Adriani, O., Barbarino, G. C., Bazilevskaya, G. A., Bellotti, R., Boezio, M., Bogomolov, E. A., Bonechi, L., Bongi, M., Bonvicini, V., Borisov, S., Bottai, S., Bruno, A., Cafagna, F., Campana, D., Carbone, R., Carlson, P., Casolino, M., Castellini, G., Consiglio, L., De Pascale, M. P., De Santis, C., De Simone, N., Di Felice, V., Galper, A. M., Gillard, W., Grishantseva, L., Jerse, G., Karelin, A. V., Koldashov, S. V., Krutkov, S. Y., Kvashnin, A. N., Leonov, A., Malakhov, V., Malvezzi, V., Marcelli, L., Mayorov, A. G., Menn, W., Mikhailov, V. V., Mocchiutti, E., Monaco, A., Mori, N., Nikonov, N., Osteria, G., Palma, F., Papini, P., Pearce, M., Picozza, P., Pizzolotto, C., Ricci, M., Ricciarini, S. B., Rossetto, L., Sarkar, R., Simon, M., Sparvoli, R., Spillantini, P., Stozhkov, Y. I., Vacchi, A., Vannuccini, E., Vasilyev, G., Voronov, S. A., Yurkin, Y. T., Wu, J., Zampa, G., Zampa, N., and Zverev, V. G.: PAMELA Measurements of Cosmic-Ray Proton and Helium Spectra, Science, 332, 69-72, doi:10.1126/science.1199172, 2011.

Aglietta, M., Alekseenko, V. V., Alessandro, B., Antonioli, P., Arneodo, F., Bergamasco, L., Bertaina, M., Bonino, R., Castellina, A., Chiavassa, A., Piazzoli, B. D., Sciascio, G. D., Fulgione, W., Galeotti, P., Ghia, P. L., Iacovacci, M., Mannocchi, G., Morello, C., Navarra, G., Saavedra, O., Stamerra, A., Trinchero, G. C., Valchierotti, S., Vallania, P., Vernetto, S., and Vigorito, C.: Evolution of the Cosmic-Ray Anisotropy Above $1014 \mathrm{eV}$, Astrophys. J. Lett., 692, L130, doi:10.1088/0004-637X/692/2/L130, 2009.

Ahn, H. S., Allison, P., Bagliesi, M. G., Beatty, J. J., Bigongiari, G., Childers, J. T., Conklin, N. B., Coutu, S., DuVernois, M. A., Ganel, O., Han, J. H., Jeon, J. A., Kim, K. C., Lee, M. H., Lutz, L., Maestro, P., Malinin, A., Marrocchesi, P. S., Minnick, S., Mognet, S. I., Nam, J., Nam, S., Nutter, S. L., Park, I. H., Park, N. H., Seo, E. S., Sina, R., Wu, J., Yang, J., Yoon, Y. S., Zei, R., and Zinn, S. Y.: Discrepant Hardening Observed in Cosmic-ray Elemental Spectra, Astrophys. J. Lett., 714, L89L93, doi:10.1088/2041-8205/714/1/L89, 2010.

Amenomori, M., Ayabe, S., Bi, X. J., Chen, D., Cui, S. W., Danzengluobu, Ding, L. K., Ding, X. H., Feng, C. F., Feng, Z., Feng, Z. Y., Gao, X. Y., Geng, Q. X., Guo, H. W., He, H. H., He, M., Hibino, K., Hotta, N., Hu, H., Hu, H. B., Huang, J., Huang, Q., Jia, H. Y., Kajino, F., Kasahara, K., Katayose, Y., Kato, C., Kawata, K., Labaciren, Le, G. M., Li, A. F., Li, J. Y., Lou, Y.-Q., Lu, H., Lu, S. L., Meng, X. R., Mizutani, K., Mu, J., Munakata, K., Nagai, A., Nanjo, H., Nishizawa, M., Ohnishi, M., Ohta, I., Onuma, H., Ouchi, T., Ozawa, S., Ren, J. R., Saito, T., Saito, T. Y., Sakata, M., Sako, T. K., Sasaki, T., Shibata, M., Shiomi, A., Shirai, T., Sugimoto, H., Takita, M., Tan, Y. H., Tateyama, N., Torii, S., Tsuchiya, H., Udo, S., Wang, B., Wang, H., Wang, X., Wang, Y. G., Wu, H. R., Xue, L., Yamamoto, Y., Yan, C. T., Yang, X. C., Yasue, S., Ye, Z. H., Yu, G. C., Yuan, A. F., Yuda, T., Zhang, H. M., Zhang, J. L., Zhang, N. J., Zhang, X. Y., Zhang, Y., 
Zhang, Y., Zhaxisangzhu, Zhou, X. X., and The Tibet AS $\gamma$ Collaboration: Anisotropy and Corotation of Galactic Cosmic Rays, Science, 314, 439-443, doi:10.1126/science.1131702, 2006.

Amenomori, M., Ayabe, S., Bi, X. J., Chen, D., Cui, S. W., Danzengluobu, Ding, L. K., Ding, X. H., Feng, C. F., Feng, Z., Feng, Z. Y., Gao, X. Y., Geng, Q. X., Guo, H. W., He, H. H., He, M., Hibino, K., Hotta, N., Hu, H., Hu, H. B., Huang, J., Huang, Q., Jia, H. Y., Kajino, F., Kasahara, K., Katayose, Y., Kato, C., Kawata, K., Labaciren, Le, G. M., Li, A. F., Li, J. Y., Lou, Y.-Q., Lu, H., Lu, S. L., Meng, X. R., Mizutani, K., Mu, J., Munakata, K., Nagai, A., Nanjo, H., Nishizawa, M., Ohnishi, M., Ohta, I., Onuma, H., Ouchi, T., Ozawa, S., Ren, J. R., Saito, T., Saito, T. Y., Sakata, M., Sako, T. K., Sasaki, T., Shibata, M., Shiomi, A., Shirai, T., Sugimoto, H., Takita, M., Tan, Y. H., Tateyama, N., Torii, S., Tsuchiya, H., Udo, S., Wang, B., Wang, H., Wang, X., Wang, Y. G., Wu, H. R., Xue, L., Yamamoto, Y., Yan, C. T., Yang, X. C., Yasue, S., Ye, Z. H., Yu, G. C., Yuan, A. F., Yuda, T., Zhang, H. M., Zhang, J. L., Zhang, N. J., Zhang, X. Y., Zhang, Y., Zhang, Y., Zhaxisangzhu, Zhou, X. X., and The Tibet AS $\gamma$ Collaboration: Implication of the sidereal anisotropy of $\sim 5 \mathrm{TeV}$ cosmic ray intensity observed with the Tibet III air shower array, in: International Cosmic Ray Conference, Vol. 1 of International Cosmic Ray Conference, Mérida, Mexico, 2007.

Amenomori, M., Ayabe, S., Bi, X. J., Chen, D., Cui, S. W., Danzengluobu, Ding, L. K., Ding, X. H., Feng, C. F., Feng, Z., Feng, Z. Y., Gao, X. Y., Geng, Q. X., Guo, H. W., He, H. H., He, M., Hibino, K., Hotta, N., Hu, H., Hu, H. B., Huang, J., Huang, Q., Jia, H. Y., Kajino, F., Kasahara, K., Katayose, Y., Kato, C., Kawata, K., Labaciren, Le, G. M., Li, A. F., Li, J. Y., Lou, Y.-Q., Lu, H., Lu, S. L., Meng, X. R., Mizutani, K., Mu, J., Munakata, K., Nagai, A., Nanjo, H., Nishizawa, M., Ohnishi, M., Ohta, I., Onuma, H., Ouchi, T., Ozawa, S., Ren, J. R., Saito, T., Saito, T. Y., Sakata, M., Sako, T. K., Sasaki, T., Shibata, M., Shiomi, A., Shirai, T., Sugimoto, H., Takita, M., Tan, Y. H., Tateyama, N., Torii, S., Tsuchiya, H., Udo, S., Wang, B., Wang, H., Wang, X., Wang, Y. G., Wu, H. R., Xue, L., Yamamoto, Y., Yan, C. T., Yang, X. C., Yasue, S., Ye, Z. H., Yu, G. C., Yuan, A. F., Yuda, T., Zhang, H. M., Zhang, J. L., Zhang, N. J., Zhang, X. Y., Zhang, Y., Zhang, Y., Zhaxisangzhu, Zhou, X. X., and The Tibet AS $\gamma$ Collaboration: Time Dependence of Loss-Cone Amplitude measured with the Tibet Air-Shower Array, in: International Cosmic Ray Conference, Vol. 1 of International Cosmic Ray Conference, Beijing China, p. 62, 2011a.

Amenomori, M., Ayabe, S., Bi, X. J., Chen, D., Cui, S. W., Danzengluobu, Ding, L. K., Ding, X. H., Feng, C. F., Feng, Z., Feng, Z. Y., Gao, X. Y., Geng, Q. X., Guo, H. W., He, H. H., He, M., Hibino, K., Hotta, N., Hu, H., Hu, H. B., Huang, J., Huang, Q., Jia, H. Y., Kajino, F., Kasahara, K., Katayose, Y., Kato, C., Kawata, K., Labaciren, Le, G. M., Li, A. F., Li, J. Y., Lou, Y.-Q., Lu, H., Lu, S. L., Meng, X. R., Mizutani, K., Mu, J., Munakata, K., Nagai, A., Nanjo, H., Nishizawa, M., Ohnishi, M., Ohta, I., Onuma, H., Ouchi, T., Ozawa, S., Ren, J. R., Saito, T., Saito, T. Y., Sakata, M., Sako, T. K., Sasaki, T., Shibata, M., Shiomi, A., Shirai, T., Sugimoto, H., Takita, M., Tan, Y. H., Tateyama, N., Torii, S., Tsuchiya, H., Udo, S., Wang, B., Wang, H., Wang, X., Wang, Y. G., Wu, H. R., Xue, L., Yamamoto, Y., Yan, C. T., Yang, X. C., Yasue, S., Ye, Z. H., Yu, G. C., Yuan, A. F., Yuda, T., Zhang, H. M., Zhang, J. L., Zhang, N. J., Zhang, X. Y., Zhang, Y., Zhang, Y., Zhaxisangzhu, Zhou, X. X., and The Tibet AS $\gamma$
Collaboration: Modeling of the galactic cosmic-ray anisotropy at $\mathrm{TeV}$ energies, in: International Cosmic Ray Conference, Vol. 1 of International Cosmic Ray Conference, Beijing, China, p. 105, 2011b.

Battaner, E., Castellano, J., and Masip, M.: Galactic Magnetic Fields and the Large-Scale Anisotropy at Milagro, Astrophys. J. Lett., 703, L90-L93, doi:10.1088/0004-637X/703/1/L90, 2009.

Bell, A. R.: The acceleration of cosmic rays in shock fronts. II, MNRAS, 182, 443-455, 1978.

Beresnyak, A., Yan, H., and Lazarian, A.: Numerical Study of Cosmic Ray Diffusion in Magnetohydrodynamic Turbulence, Astrophys. J., 728, 60-+, doi:10.1088/0004-637X/728/1/60, 2011.

Birn, J., Thomsen, M. F., and Hesse, M.: Electron acceleration in the dynamic magnetotail: Test particle orbits in three-dimensional magnetohydrodynamic simulation fields, Phys. Plasmas, 11, 1825-1833, doi:10.1063/1.1704641, 2004.

Biskamp, D.: Magnetic Reconnection in Plasmas, Astrophys. Space Sci., 242, 165-207, doi:10.1007/BF00645113, 1996.

Blasi, P. and Amato, E.: Diffusive propagation of cosmic rays from supernova remnants in the Galaxy. II: anisotropy, JCAP, 1, 11, doi:10.1088/1475-7516/2012/01/011, 2012.

Ciaravella, A. and Raymond, J. C.: The Current Sheet Associated with the 2003 November 4 Coronal Mass Ejection: Density, Temperature, Thickness, and Line Width, Astrophys. J., 686, 1372 1382, doi:10.1086/590655, 2008.

Compton, A. H. and Getting, I. A.: An Apparent Effect of Galactic Rotation on the Intensity of Cosmic Rays, Phys. Rev., 47, 817821, doi:10.1103/PhysRev.47.817, 1935.

de Gouveia Dal Pino, E. M. and Lazarian, A.: The role of Violent Magnetic Reconnection on the Production of the Large Scale Superluminal Ejections of the Microquasar GRS 1915+105, arXiv:astro-ph/0307054, 2003.

de Gouveia Dal Pino, E. M. and Lazarian, A.: Production of the large scale superluminal ejections of the microquasar GRS $1915+105$ by violent magnetic reconnection, A\&A, 441, 845853, doi:10.1051/0004-6361:20042590, 2005.

Desiati, P. and Lazarian, A.: Anisotropy of $\mathrm{TeV}$ Cosmic Rays and the Outer Heliospheric Boundaries, ArXiv e-prints, arXiv:1111.3075, 2011.

Di Sciascio, G. and the ARGO-YBJ Collaboration: Measurement of Cosmic Ray spectrum and Anisotropy with ARGO-YBJ, ArXiv e-prints, arXiv:1202.3379, 2012.

Drake, J. F., Swisdak, M., Che, H., and Shay, M. A.: Electron acceleration from contracting magnetic islands during reconnection, Nature, 443, 553-556, doi:10.1038/nature05116, 2006.

Drake, J. F., Opher, M., Swisdak, M., and Chamoun, J. N.: A Magnetic Reconnection Mechanism for the Generation of Anomalous Cosmic Rays, Astrophys. J., 709, 963-974, doi:10.1088/0004637X/709/2/963, 2010.

Drury, L. O. and Aharonian, F. A.: The puzzling MILAGRO hot spots, Astropart. Phys., 29, 420-423, doi:10.1016/j.astropartphys.2008.04.007, 2008.

Erlykin, A. D. and Wolfendale, A. W.: The anisotropy of galactic cosmic rays as a product of stochastic supernova explosions, Astropart. Phys., 25, 183-194, doi:10.1016/j.astropartphys.2006.01.003, 2006.

Frisch, P. C.: How Local is the Local Interstellar Magnetic Field?, ArXiv e-prints, arXiv:1111.3675, 2011. 
Frisch, P. C., Redfield, S., and Slavin, J. D.: The Interstellar Medium Surrounding the Sun, Annu. Rev. Astro. Astrophys., 49, 237279, doi:10.1146/annurev-astro-081710-102613, 2011.

Giacinti, G. and Sigl, G.: Local Magnetic Turbulence and TeVPeV Cosmic Ray Anisotropies, ArXiv e-prints, arXiv:1111.2536, 2011.

Gieseler, U. D. J. and Jones, T. W.: First order Fermi acceleration at multiple oblique shocks, A\&A, 357, 1133-1136, 2000.

Gleeson, L. J. and Axford, W. I.: The Compton-Getting Effect, Astrophys. Space Sci., 2, 431-437, doi:10.1007/BF02175919, 1968.

Guillian, G., Hosaka, J., Ishihara, K., Kameda, J., Koshio, Y., Minamino, A., Mitsuda, C., Miura, M., Moriyama, S., Nakahata, M., Namba, T., Obayashi, Y., Ogawa, H., Shiozawa, M., Suzuki, Y., Takeda, A., Takeuchi, Y., Yamada, S., Higuchi, I., Ishitsuka, M., Kajita, T., Kaneyuki, K., Mitsuka, G., Nakayama, S., Nishino, H., Okada, A., Okumura, K., Saji, C., Takenaga, Y., Desai, S., Kearns, E., Stone, J. L., Sulak, L. R., Wang, W., Goldhaber, M., Casper, D., Gajewski, W., Griskevich, J., Kropp, W. R., Liu, D. W., Mine, S., Smy, M. B., Sobel, H. W., Vagins, M. R., Ganezer, K. S., Hill, J., Keig, W. E., Scholberg, K., Walter, C. W., Ellsworth, R. W., Tasaka, S., Kibayashi, A., Learned, J. G., Matsuno, S., Messier, M. D., Hayato, Y., Ichikawa, A. K., Ishida, T., Ishii, T., Iwashita, T., Kobayashi, T., Nakadaira, T., Nakamura, K., Nitta, K., Oyama, Y., Totsuka, Y., Suzuki, A. T., Hasegawa, M., Kato, I., Maesaka, H., Nakaya, T., Nishikawa, K., Sato, H., Yamamoto, S., Yokoyama, M., Haines, T. J., Dazeley, S., Hatakeyama, S., Svoboda, R., Blaufuss, E., Goodman, J. A., Sullivan, G. W., Turcan, D., Habig, A., Fukuda, Y., Itow, Y., Sakuda, M., Yoshida, M., Kim, S. B., Yoo, J., Okazawa, H., Ishizuka, T., Jung, C. K., Kato, T., Kobayashi, K., Malek, M., Mauger, C., McGrew, C., Sharkey, E., Yanagisawa, C., Gando, Y., Hasegawa, T., Inoue, K., Shirai, J., Suzuki, A., Nishijima, K., Ishino, H., Watanabe, Y., Koshiba, M., Kielczewska, D., Berns, H. G., Gran, R., Shiraishi, K. K., Stachyra, A. L., Washburn, K., Wilkes, R. J., and Munakata, K.: Observation of the anisotropy of $10 \mathrm{TeV}$ primary cosmic ray nuclei flux with the Super-Kamiokande-I detector, Phys. Rev. D, 75, 062003, doi:10.1103/PhysRevD.75.062003, 2007.

Iuppa, R.: Few-degree anisotropies in the cosmic-ray flux observed by the ARGO-YBJ experiment, in: International Cosmic Ray Conference, Vol. 1 of International Cosmic Ray Conference, Beijing China, p. 74, 2011.

Izmodenov, V. V. and Alexashov, D. B.: A Model for the Tail Region of the Heliospheric Interface, Astron. Lett., 29, 58-63, doi:10.1134/1.1537379, 2003.

Izmodenov, V. V. and Kallenbach, R. (Eds.): The Physics of the Heliospheric Boundaries, 2006.

Kliem, B.: Particle orbits, trapping, and acceleration in a filamentary current sheet model, Astrophys. J. Suppl., 90, 719-728, doi:10.1086/191896, 1994.

Kowal, G., Lazarian, A., Vishniac, E. T., and Otmianowska-Mazur, K.: Numerical Tests of Fast Reconnection in Weakly Stochastic Magnetic Fields, Astrophys. J., 700, 63-85, doi:10.1088/0004637X/700/1/63, 2009.

Kowal, G., de Gouveia Dal Pino, E. M., and Lazarian, A.: Magnetohydrodynamic Simulations of Reconnection and Particle Acceleration: Three-dimensional Effects, Astrophys. J., 735, 102, doi:10.1088/0004-637X/735/2/102, 2011.
Kowal, G., de Gouveia Dal Pino, E. M., and Lazarian, A.: Particle Acceleration in Turbulence and Weakly Stochastic Reconnection, PRL, accepted, 2012.

Lazarian, A.: Magnetic Fields in the Universe: From Laboratory and Stars to Primordial Structures, in: Magnetic Fields in the Universe: From Laboratory and Stars to Primordial Structures, edited by: de Gouveia Dal Pino, E. M., Lugones, G., and Lazarian, A., vol. 784 of American Institute of Physics Conference Series, 2005.

Lazarian, A.: Enhancement and Suppression of Heat Transfer by MHD Turbulence, Astrophys. J. Lett., 645, L25-L28, doi:10.1086/505796, 2006.

Lazarian, A.: Erratum: "Enhancement and Suppression of Heat Transfer by MHD Turbulence", Astrophys. J. Lett., 660, L173L173, doi:10.1086/518163, 2007.

Lazarian, A. and Desiati, P.: Magnetic Reconnection as the Cause of Cosmic Ray Excess from the Heliospheric Tail, Astrophys. J., 722, 188-196, doi:10.1088/0004-637X/722/1/188, 2010.

Lazarian, A. and Opher, M.: A Model of Acceleration of Anomalous Cosmic Rays by Reconnection in the Heliosheath, Astrophys. J., 703, 8-21, doi:10.1088/0004-637X/703/1/8, 2009.

Lazarian, A. and Vishniac, E. T.: Reconnection in a Weakly Stochastic Field, Astrophys. J., 517, 700-718, doi:10.1086/307233, 1999.

Lazarian, A., Vishniac, E. T., and Cho, J.: Magnetic Field Structure and Stochastic Reconnection in a Partially Ionized Gas, Astrophys. J., 603, 180-197, doi:10.1086/381383, 2004.

Lazarian, A., Kowal, G., Vishniac, E., and de Gouveia Dal Pino, E.: Fast magnetic reconnection and energetic particle acceleration, Planet. Space Sci., 59, 537-546, doi:10.1016/j.pss.2010.07.020, 2011.

Liewer, P. C., Karmesin, S. R., and Brackbill, J. U.: Hydrodynamic instability of the heliopause driven by plasma-neutral chargeexchange interactions, J. of Geophys. Res., 101, 17119-17128, doi:10.1029/96JA00606, 1996.

Longair, M. S.: High energy astrophysics, Vol. 2, 1992.

Malkov, M. A., Diamond, P. H., O'C. Drury, L., and Sagdeev, R. Z.: Probing Nearby Cosmic-ray Accelerators and Interstellar Medium Turbulence with MILAGRO Hot Spots, Astrophys. J., 721, 750-761, doi:10.1088/0004-637X/721/1/750, 2010.

Matthaeus, W. H., Ambrosiano, J. J., and Goldstein, M. L.: Particle-acceleration by turbulent magnetohydrodynamic reconnection, Phys. Rev. Lett., 53, 1449-1452, doi:10.1103/PhysRevLett.53.1449, 1984.

Melrose, D. B. and Pope, M. H.: Diffusive Shock Acceleration by Multiple Shocks, Proc. Astronom. Soc. Australia, 10, 222, 1993.

Munakata, K., Mizoguchi, Y., Kato, C., Yasue, S., Mori, S., Takita, M., and Kóta, J.: Solar Cycle Dependence of the Diurnal Anisotropy of $0.6 \mathrm{TeV}$ Cosmic-ray Intensity Observed with the Matsushiro Underground Muon Detector, Astrophys. J., 712, 1100, doi:10.1088/0004-637X/712/2/1100, 2010.

Nagashima, K., Fujimoto, K., and Jacklyn, R. M.: Galactic and heliotail-in anisotropies of cosmic rays as the origin of sidereal daily variation in the energy region $; 10^{4} \mathrm{GeV}$, J. Geophys. Res., 1031, 17429-17440, doi:10.1029/98JA01105, 1998.

Nerney, S., Suess, S. T., and Schmahl, E. J.: Flow downstream of the heliospheric terminal shock: Magnetic field line topology and solar cycle imprint, J. Geophys. Res., 100, 3463-3471, doi:10.1029/94JA02690, 1995. 
Parker, E. N.: Sweet's Mechanism for Merging Magnetic Fields in Conducting Fluids, J. Geophys. Res., 62, 509-520, doi:10.1029/JZ062i004p00509, 1957.

Parker, E. N.: Kinetic properties of interplanetary matter, Planet. Space Sci., 9, 461-475, doi:10.1016/0032-0633(62)90050-8, 1962.

Parker, E. N.: The passage of energetic charged particles through interplanetary space, Planet. Space Sci., 13, 461-475, doi:10.1016/0032-0633(65)90131-5, 1965.

Parker, E. N.: The Generation of Magnetic Fields in Astrophysical Bodies. I. The Dynamo Equations, Astrophys. J., 162, 665, doi:10.1086/150697, 1970.

Parker, E. N.: Cosmical magnetic fields: Their origin and their activity, 1979.

Petschek, H. E.: Magnetic Field Annihilation, NASA Special Publication, 50, 425, 1964.

Pogorelov, N. V., Borovikov, S. N., Zank, G. P., and Ogino, T.: Three-Dimensional Features of the Outer Heliosphere Due to Coupling Between the Interstellar and Interplanetary Magnetic Fields, III. The Effects of Solar Rotation and Activity Cycle, Astrophys. J., 696, 1478-1490, doi:10.1088/0004637X/696/2/1478, 2009a.

Pogorelov, N. V., Heerikhuisen, J., Zank, G. P., Mitchell, J. J., and Cairns, I. H.: Heliospheric asymmetries due to the action of the interstellar magnetic field, Adv. Space Res., 44, 1337-1344, doi:10.1016/j.asr.2009.07.019, 2009b.

Salvati, M.: The local Galactic magnetic field in the direction of Geminga, A\&A, 513, A28, doi:10.1051/0004-6361/200913406, 2010.

Salvati, M. and Sacco, B.: The Milagro anticenter hot spots: cosmic rays from the Geminga supernova?, A\&A, 485, 527-529, doi:10.1051/0004-6361:200809586, 2008.

Scherer, K. and Fahr, H. J.: Breathing of heliospheric structures triggered by the solar-cycle activity, Ann. Geophys., 21, 1303-1313, doi:10.5194/angeo-21-1303-2003, 2003.

Shaikh, D. and Zank, G. P.: Modulation of waves due to charge-exchange collisions in magnetized partially ionized space plasma, Phys. Lett. A, 374, 4538-4542, doi:10.1016/j.physleta.2010.09.020, 2010.

Shay, M. A. and Drake, J. F.: The role of electron dissipation on the rate of collisionless magnetic reconnection, Geophys. Res. Lett., 25, 3759-3762, doi:10.1029/1998GL900036, 1998.
Shay, M. A., Drake, J. F., Denton, R. E., and Biskamp, D.: Structure of the dissipation region during collisionless magnetic reconnection, J. Geophys. Res., 103, 9165-9176, doi:10.1029/97JA03528, 1998.

Shay, M. A., Drake, J. F., Swisdak, M., and Rogers, B. N.: The scaling of embedded collisionless reconnection, Phys. Plasmas, 11, 2199-2213, doi:10.1063/1.1705650, 2004.

Shuwang, C.: Study on large-scale CR anisotropy with ARGO-YBJ experiment, in: International Cosmic Ray Conference, Vol. 1 of International Cosmic Ray Conference, Beijing China, p. 6, 2011.

Sturrock, P. A.: Model of the High-Energy Phase of Solar Flares, Nature, 211, 695-697, doi:10.1038/211695a0, 1966.

Sweet, P. A.: The Neutral Point Theory of Solar Flares, in: Electromagnetic Phenomena in Cosmical Physics, edited by B. Lehnert, Vol. 6 of IAU Symposium, p. 123, 1958.

Vernetto, S., Guglielmotto, Z., Zhang, J. L., and the ARGOYBJ Collaboration: Sky monitoring with ARGO-YBJ, arXiv:0907.4615, 2009.

Wefel, J. P., Adams, Jr., J. H., Ahn, H. S., and et al.: Revised Energy Spectra for Primary Elements $(\mathrm{H}-\mathrm{Si}$ ) above $50 \mathrm{GeV}$ from the ATIC-2 Science Flight, in: International Cosmic Ray Conference, Vol. 2 of International Cosmic Ray Conference, 31-34, 2008.

Wygant, J. R., Cattell, C. A., Lysak, R., Song, Y., Dombeck, J., McFadden, J., Mozer, F. S., Carlson, C. W., Parks, G., Lucek, E. A., Balogh, A., Andre, M., Reme, H., Hesse, M., and Mouikis, C.: Cluster observations of an intense normal component of the electric field at a thin reconnecting current sheet in the tail and its role in the shock-like acceleration of the ion fluid into the separatrix region, J. Geophys. Res., 110, A09206, doi:10.1029/2004JA010708, 2005.

Yan, H. and Lazarian, A.: Cosmic-Ray Scattering and Streaming in Compressible Magnetohydrodynamic Turbulence, Astrophys. J., 614, 757-769, doi:10.1086/423733, 2004.

Yan, H. and Lazarian, A.: Cosmic-Ray Propagation: Nonlinear Diffusion Parallel and Perpendicular to Mean Magnetic Field, Astrophys. J., 673, 942-953, doi:10.1086/524771, 2008.

Zhang, J. L.: Observation of $\mathrm{TeV}$ cosmic ray anisotropy by the ARGO-YBJ, in: International Cosmic Ray Conference, Vol. 1 of International Cosmic Ray Conference, Łódź, Poland, 2009. 